\title{
Short communication: Physicochemical features and microbial community of milk kefir using a potential probiotic Saccharomyces cerevisiae KU200284
}

\author{
Ji-Young Hong, ${ }^{1,2}$ Na-Kyoung Lee, ${ }^{1}$ Sung-Hun $\mathrm{Yi}^{2}{ }^{2}$ Sang-Pil Hong, ${ }^{2}$ and Hyun-Dong Paik ${ }^{1 *} \odot$ \\ ${ }^{1}$ Department of Food Science and Biotechnology of Animal Resources, Konkuk University, Seoul 05029, Korea \\ ${ }^{2}$ Research Group of Traditional Food, Korea Food Research Institute, Wanju 55365, Korea
}

\section{ABSTRACT}

The aim of this study was to analyze the $\beta$-glucan contents, physicochemical features, and microbial communities in milk kefir prepared using Saccharomyces cerevisiae KU200284 isolated from cucumber jangajji, a fermented vegetable commonly eaten in Korean. Three types of milk kefir were manufactured, with (1) activated kefir grain, (2) activated kefir grain with commercial S. cerevisiae BOF, and (3) activated kefir grain with $S$. cerevisiae KU200284. $\beta$-Glucan contents of milk kefir using kefir grain and kefir grain with $S$. cerevisiae strains BOF and KU200284 were 8.29, 8.59, and $8.57 \%$, respectively. The $\mathrm{pH}$, titratable acidity, viscosity, Brix level, and alcohol contents of milk kefir using kefir grain with $S$. cerevisiae strains were acceptable compared with milk kefir using only kefir grain. In milk kefir produced using kefir grains and $S$. cerevisiae strains, $16 \mathrm{~S}$ rRNA reads showed representative strains of Lactobacillus kefiranofaciens ( $>72 \%$ relative abundance) and Acetobacter fabarum ( $>16 \%$ relative abundance). In particular, milk kefir using kefir grain with S. cerevisiae KU200284 had the highest relative abundance of L. kefiranofaciens. In addition, the internal transcribed sequence (ITS) rRNA reads in tested milk kefir showed representative strains of Kluyveromyces marxianus ( $>52 \%$ relative abundance) and Saccharomyces cerevisiae ( $>16 \%$ relative abundance). In contrast, milk kefir using $S$. cerevisiae strains had higher relative abundance of $S$. cerevisiae $(>37 \%)$. The $\beta$-glucan production, physicochemical properties, and microbial community profiling indicate that $S$. cerevisiae KU200284 could be used in functional dairy products as a starter culture.

Key words: Saccharomyces cerevisiae, milk kefir, physicochemical feature, microbial community

Received January 28, 2019.

Accepted July 17, 2019.

*Corresponding author: hdpaik@konkuk.ac.kr

\section{Short Communication}

Kefir is a fermented dairy beverage made from kefir grains, including lactic acid bacteria, yeasts, and acetic acid bacteria (Garofalo et al., 2015). Kefir grains are elastic, slimy, white to light yellow, and with a small, irregular, round shape, often differing in size, and sometimes resembling a mushroom (Kabak and Dobson, 2011). In general, kefir grains are composed of Kluyveromyces, Saccharomyces, Lactobacillus, Lactococcus, Leuconostoc, and Acetobacter species (Prado et al., 2015). Kefir is obtained through the lactic or alcoholic fermentation of milk, and their microbiota produces bioactive compounds, including peptides, amino acids, ethanol, $\mathrm{CO}_{2}$, acetaldehyde, acetoin, diacetyl, exopolysaccharides, folic acid, calcium, vitamins $\left(\mathrm{B}_{1}, \mathrm{~B}_{12}\right.$, and K) (Kabak and Dobson, 2011; Garofalo et al., 2015). Functional effects have been also reported, including immune system modulation, enhanced digestive health, and antimicrobial, antitumor, and antioxidant activities (Farnworth, 2005).

Saccharomyces cerevisiae has been classified as QPS (qualified presumption of safety) according to the European Food Safety Authority (Leuschner et al., 2010). Saccharomyces cerevisiae has been used in winemaking, baking, and brewing, with reported functions including the production of $\beta$-glucan, glutathione, glutathione derivatives, protein, fiber, vitamin $\mathrm{B}$, and folic acid derived from cell wall (Hong et al., 2019). Saccharomyces cerevisiae or Saccharomyces boulardii has mainly been used as a probiotic for treatment of antibiotic-associated diarrhea, and studies have indicated that either species can perform immunomodulatory, gastrointestinal modulatory, and antioxidant functions (Fakruddin et al., 2017). Saccharomyces cerevisiae KU200284 was previously isolated from cucumber jangajji, a Korean vegetable fermented food. This strain was demonstrated to have the highest $\beta$-glucan production, stability in artificial gastric conditions, without transfer of antibiotic resistance genes, and protection against DNA damage among the tested S. cerevisiae strains (Lee et al., 2019). In addition, $S$. cerevisiae KU200284 demonstrated the highest adhesion to intestinal cells. Therefore, we made 
milk kefirs using kefir grains, kefir grains with $S$. cerevisiae BOF, and kefir grains with $S$. cerevisiae KU200284, and evaluated their physicochemical features. In addition, we performed a next-generation sequencing-based microbiome analysis to assess the microbial community composition of milk kefir inoculating potential probiotic yeast strains.

We used S. cerevisiae KU200284 or S. cerevisiae $\mathrm{BOF}$ isolated from a pharmaceutical product (Biocodex, Gentilly, France) as starter culture supplement. Yeast strains were cultured in yeast malt (YM) medium (Difco, Detroit, MI) at $25^{\circ} \mathrm{C}$ for $48 \mathrm{~h}$ for further study.

The kefir grains were obtained from a local household (Lyosan, Canada). Activation of kefir grains followed the method of Leite et al. (2013) with modification. Two grams of kefir grains was used to inoculate 100 $\mathrm{mL}$ of sterilized milk and then incubated at $26^{\circ} \mathrm{C}$ for 24 h. After incubation, the culture was filtered through a plastic strainer to remove coagulated milk and gently washed with sterilized water. This step was repeated 4 times until the kefir grains had appropriate characteristics and their biomass had increased by $10 \%$.

Three types of milk kefir were manufactured using activated kefir grains. Milk kefir was inoculated with $2 \%$ (wt/vol) activated kefir grains into sterilized whole milk (M1). Sterilized whole milk was inoculated with $2 \%$ activated kefir grains and $6 \log \mathrm{cfu} / \mathrm{mL}$ of either $S$. cerevisiae BOF (M2) or S. cerevisiae KU200284 (M3). The inoculated milk was fermented at $26^{\circ} \mathrm{C}$ for $24 \mathrm{~h}$, and sampling was performed at $0,8,16$, and $24 \mathrm{~h}$ for physicochemical characterization.

At $24 \mathrm{~h}$ of sampling, the milk kefirs were freeze-dried, and dried samples were quantified using the Megazyme kit (Megazyme Inc., Wicklow, Ireland) following the manufacturer's guideline for glucan calculation.

The $\mathrm{pH}$ of milk kefir samples was measured using a pH meter (SevenCompact, Mettler Toledo, Greifensee, Switzerland), and titratable acidity was measured using a titrator (848 Titrino Plus, Metrohm, Herisau, Switzerland). The viscosity of kefir samples was measured 3 times at 1-min intervals from 2 to 4 min at 30 rpm, using a DV-E viscometer (Brookfield Engineering Laboratories Inc., Middleboro, MA) 64 spindle. The Brix values of kefir samples were measured using a Brix meter (PR-1, Atago, Tokyo, Japan) in the range of 0 to $32 \%$ Brix. The alcohol contents of kefir beverages were measured using a concentration meter (DMA 4500A, Anton Paar GmbH, Graz, Austria).

For microbial composition, total DNA was extracted from $30 \mathrm{~mL}$ of milk kefir after $24 \mathrm{~h}$ of fermentation, using a PowerSoil DNA isolation kit (MO BIO Laboratories Inc., Carlsbad, CA) according to the manufacturer's protocol. The DNA quality was measured by PicoGreen dye (Invitrogen, Carlsbad, CA) and Nanodrop 2000 spectrophotometer (Thermo Fisher Scientific, Wilmington, DE). The V3-V4 regions of bacterial 16S rRNA genes were amplified by PCR from the genomic DNA (forward primer: 5'-TCGTCGGCAGCGTCAGATGTGTATAAGGACAGCCTACGGGNGGCWGCAG-3' reverse primer: 5'-GGCTCGGATGTGTATAGAACAGGACTACHVGGGTATCTAATCC-3'). The internal transcribed sequence (ITS) rRNA genes were used for fungal microbiome analysis (forward primer: 5'-GCATCGATGAAGAACGCAGC-3'; reverse primer: $5^{\prime}$-TCCTCCGCTTATTGTATGC-3'). The final products were normalized and pooled using the PicoGreen kit. Library size was verified using the LabChip GX HT DNA high sensitivity kit (PerkinElmer, Waltham, MA). Sequence processing was performed using the Illumina MiSeq platform (Illumina Inc., San Diego, CA). Sequence data were trimmed using the FASTX tool (v 0.0.14) and analyzed with QIIME 1.8.0 (Caporaso et al., 2010). The sequences were clustered into operational taxonomic units (OTU) using the default UCLUST closed-reference OTU picking algorithm (pick_closed_referne_otus.py) against the curated sequences (Kim et al., 2019); OTU represented $>0.5 \%$ of the total sequences.

The results are expressed as mean and standard deviation of 3 replicates in triplicate. One-way ANOVA was used to determine significant differences. Comparison of means was carried out using Duncan's multiple range test $(P<0.05)$.

Saccharomyces cerevisiae KU200284 was selected as a kefir starter with a view to its production of bioactive compounds and potential probiotic properties (Lee et al., 2019). The $\beta$-glucan contents of M1, M2, and M3 were $8.29,8.59$, and $8.57 \%$, respectively (Table 1 ), and did not differ significantly between samples. However, kefir with S. cerevisiae strains (M2 and M3) showed numerically higher $\beta$-glucan contents. The total glucan contents of samples M1, M2, and M3 were 9.18, 9.18, and $9.46 \%$, respectively (Table 1 ).

Changes in $\mathrm{pH}$ and titratable acidity values of milk kefirs during fermentation are shown in Figure 1A. Ini-

Table 1. Glucan contents of the milk kefir ${ }^{1,2}$

\begin{tabular}{lccc}
\hline Item & M1 & M2 & M3 \\
\hline Total glucan (\%) & $9.18 \pm 0.99^{\mathrm{b}}$ & $9.18 \pm 0.50^{\mathrm{ab}}$ & $9.46 \pm 0.00^{\mathrm{a}}$ \\
$\alpha$-Glucan (\%) & $0.89 \pm 0.00^{\mathrm{a}}$ & $0.59 \pm 0.51^{\mathrm{a}}$ & $0.89 \pm 0.00^{\mathrm{a}}$ \\
$\beta$-Glucan (\%) & $8.29 \pm 1.00^{\mathrm{b}}$ & $8.59 \pm 0.02^{\mathrm{a}}$ & $8.57 \pm 0.00^{\mathrm{a}}$ \\
\hline
\end{tabular}

${ }^{\mathrm{a}, \mathrm{b}}$ Means with different superscript letters within a row indicate statistical differences $(P<0.05)$.

${ }^{1}$ Results are represented as mean $\pm \mathrm{SD}$.

${ }^{2} \mathrm{M} 1=$ kefir grains $(2 \%) ; \mathrm{M} 2=$ kefir grains $(2 \%)+$ Saccharomyces cerevisiae BOF; M3 = kefir grains $(2 \%)+$ Saccharomyces cerevisiae KU200284. 
tial $\mathrm{pH}$ of milk kefir ranged from 6.68 to 6.73 , and final $\mathrm{pH}$ ranged from 4.90 to 4.16 at $24 \mathrm{~h}$ of fermentation. In contrast, acidity increased from 0.13 to $1.60 \%$ during the 24-h fermentation. Generally, kefir beverages reach a pH of 4.4 to 4.6 (Wszolek et al., 2001) and acidity of 0.97 to $1.40 \%$ (Kim and Cho, 2009).

Changes in viscosity and Brix values of milk kefir during fermentation are shown in Figure 1C and 1D.
All samples showed similar viscosity and Brix values to those at $8 \mathrm{~h}$ of fermentation. At $24 \mathrm{~h}, \mathrm{M} 2 \mathrm{had}$ the lowest viscosity of 1,212 $\mathrm{cP}$, and M1 the highest viscosity of 1,948 cP; M2 showed the lowest Brix value and M1 the highest Brix value.

Changes in alcohol contents of milk kefir during fermentation are shown in Figure 1E. All samples showed $0 \%$ until 8 h; M1, M2, and M3 showed 0.29, 0.64, and

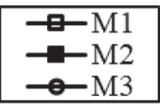

(A)

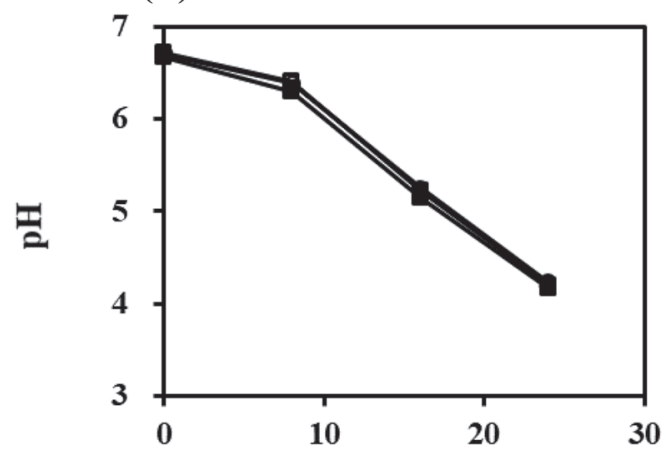

(C)

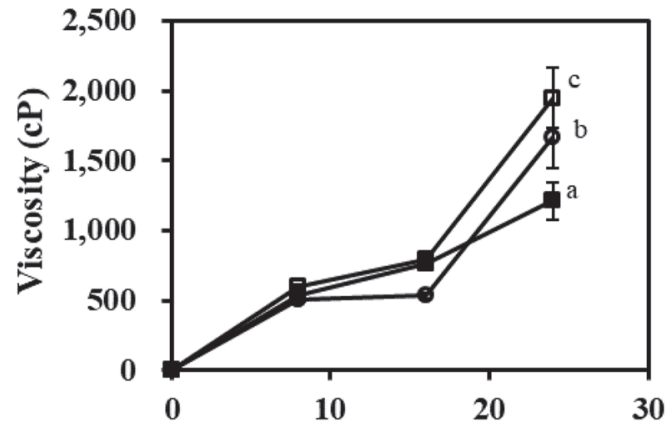

(E)

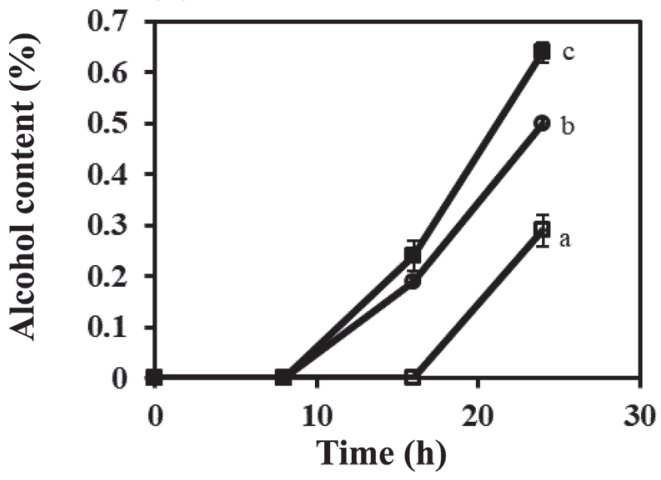

(B)

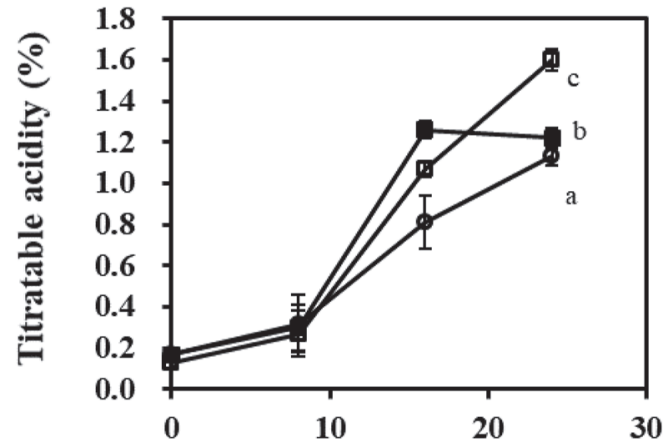

(D)

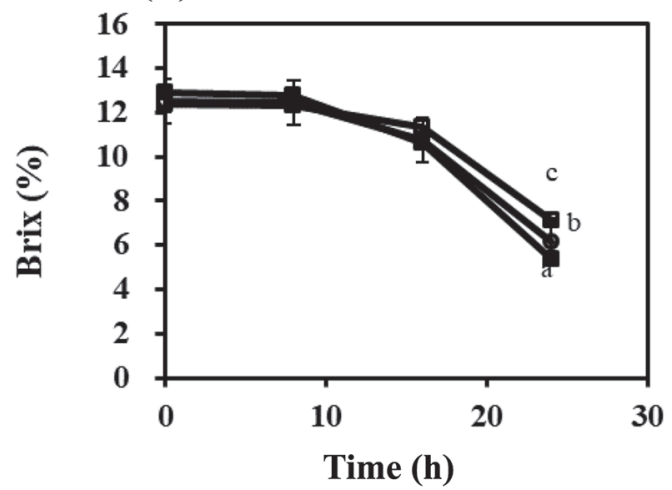

Figure 1. Changes in (A) pH, (B) titratable acidity, (C) viscosity, (D) Brix, and (E) alcohol content in kefir beverages made with kefir grains (M1; 2\%); kefir grains (2\%) + Saccharomyces cerevisiae BOF (M2); kefir grains (2\%) + Saccharomyces cerevisiae KU200284 (M3). Different letters $(\mathrm{a}-\mathrm{c})$ represent significant differences between values $(P<0.05)$. Results are presented as mean $\pm \mathrm{SD}$. 
$0.5 \%$ alcohol at $24 \mathrm{~h}$ of fermentation, respectively. The difference in alcohol contents was dependent on inoculated S. cerevisiae strains. The alcohol contents of kefir beverages are reported to vary from 0.5 to $1.5 \%$ (Marshall and Cole, 1985). Alcoholic fermented milk has been reported as a high-value functional food, with functions such as inhibition of intestinal harmful bacteria and flavor (Prado et al., 2015).

Kefir beverage is a natural probiotic carrier, consumed for its positive healthy effects (Koh et al., 2017). Therefore, the microbial community of kefir was determined using $16 \mathrm{~S}$ rRNA and ITS sequencing. In the $16 \mathrm{~S}$ rRNA sequence, read counts of M1, M2, and M3 milk kefir were $83,871,89,673$, and 111,114 . The number of observed OTU, evaluated on a $97 \%$ similarity threshold by the Chao1 estimator, ranged from 11 to 12 OTU. The rarefaction curves of observed species richness reached a saturation phase, with a $97 \%$ sequence similarity cut-off. The Shannon diversity and Simpson's indices ranged from 0.94 to 1.07 and 0.36 to 0.42 , respectively. Good's coverage was estimated to evaluate the completeness of sampling, and samples M1, M2, and M3 had values of $0.99,0.99$, and 1.00 , respectively.

The $16 \mathrm{~S}$ rRNA reads from all samples were assigned to 7 species, including Lactobacillus kefiranofaciens and Acetobacter fabarum (Table 2). The sample of M1 contained L. kefiranofaciens (76.65\%), A.fabarum (19.94\%), and Lactobacillus kefiri (3.11\%); M2 contained L. kefiranofaciens $(72.70 \%)$, A. fabarum $(22.07 \%)$, and L. kefiri (4.85\%); M3 contained L. kefiranofaciens (78.34\%), A. fabarum (16.91\%), and L. kefiri (4.51\%). Lactobacillus kefiranofaciens has a reported abundance of $97.63 \%$ in Turkish kefir grains using metagenomic analysis (Nalbantoglu et al., 2014). Lactobacillus kefiranofaciens produced "kefiran (water-soluble polysaccharide)" (Wang et al., 2012; Garofalo et al., 2015). In addition, Lactobacillus strains showed the highest abundance in M3 milk kefir at the genus level. Lactobacillus buchneri, Lactobacillus crispatus, and Lactobacillus psittaci were identified as minor microorganisms of milk kefir.

For the ITS rRNA sequence, each sequence read of M1, M2, and M3 milk kefirs was 163,775, 190,149, and 191,918, respectively (data not shown). For ITS rRNA reads, the numbers of observed OTU were 9,8 , and 47 , respectively, in M1, M2, and M3. The Shannon diversity and Simpson's indices ranged from 1.12 to 1.24 and 0.43 to 0.53 , respectively. Good's coverage of samples M1, M2, and M3 were 1.00, 0.99, and 0.99, respectively.

The ITS rRNA reads from all samples were assigned to 19 species, including Kluyveromyces marxianus and $S$. cerevisiae (Table 2); K. marxianus was dominant in all samples, particularly M1, where it was present at $72.559 \%$. Kefir samples M2 and M3 contained 52.155 and $57.390 \%$, respectively. The relative abundance of $S$. cerevisiae was higher in M2. Kluyveromyces marxianus has been reported to be the dominant yeast in Chinese koumiss and Lactobacillus fermented milk ( $\mathrm{Ni}$ et al., 2007; Zhang et al., 2017). A mixed culture of K. marxianus produced bioactive peptides with antibacterial, antihypertensive, and other properties (Zhang et al., 2017). The samples of M2 and M3 contained a higher $S$. cerevisiae proportion than M1, which was not inoculated with $S$. cerevisiae.

In this study, we produced milk kefir using potential probiotic strain S. cerevisiae KU200284. In terms of physicochemical features, the addition of $S$. cerevisiae decreased viscosity, Brix values, and total acidity and increased glucan and alcohol contents. In the microbial community, L. kefiranofaciens, A. fabarum, $K$. marxianus, and $S$. cerevisiae were dominant in all milk kefir samples. These strains could have important roles during kefir fermentation. In particular, M3 kefir had the highest abundance of Lactobacillus spp. and Saccharomyces spp. of the tested milk kefir. Therefore, $S$. cerevisiae KU200284 could be used as a starter culture for kefir production using a probiotic carrier.

Table 2. Relative abundance analysis by $16 \mathrm{~S}$ rRNA and internal transcribed spacer (ITS) rRNA sequence

\begin{tabular}{|c|c|c|c|}
\hline \multirow[b]{2}{*}{ Taxon } & \multicolumn{3}{|c|}{ Relative abundance $^{1}(\%)$} \\
\hline & M1 & M2 & M3 \\
\hline \multicolumn{4}{|l|}{ 16S rRNA sequence } \\
\hline Lactobacillus kefiranofaciens & 76.646 & 72.696 & 78.343 \\
\hline Acetobacter fabarum & 19.935 & 22.072 & 16.908 \\
\hline Lactobacillus kefiri & 3.113 & 4.851 & 4.505 \\
\hline Gluconobacter cerevisiae & 0.207 & 0.101 & 0.081 \\
\hline Lactobacillus buchneri & 0.063 & 0.101 & 0.081 \\
\hline Lactobacillus crispatus & 0.020 & 0.023 & 0.018 \\
\hline Lactobacillus psittaci & 0.010 & 0.004 & 0.012 \\
\hline \multicolumn{4}{|l|}{ ITS rRNA sequence } \\
\hline Kluyveromyces marxianus & 72.559 & 52.155 & 57.390 \\
\hline Saccharomyces cerevisiae & 16.669 & 44.431 & 37.486 \\
\hline Other & 10.687 & 3.387 & 4.825 \\
\hline Penicillium sp. & 0.001 & 0.002 & 0.079 \\
\hline Fungi & 0.000 & 0.000 & 0.007 \\
\hline Dokmaia sp. & 0.000 & 0.000 & 0.013 \\
\hline Periconia macrospinosa & 0.000 & 0.000 & 0.008 \\
\hline Saitozyma podzolica & 0.000 & 0.000 & 0.006 \\
\hline Chaetothyriales sp. & 0.000 & 0.000 & 0.018 \\
\hline Bipolaris sorokiniana & 0.000 & 0.000 & 0.002 \\
\hline Aspergillus sp. & 0.001 & 0.001 & 0.004 \\
\hline Capnodiales sp. & 0.000 & 0.000 & 0.002 \\
\hline Drechslera salviniae & 0.000 & 0.000 & 0.003 \\
\hline Helotiales sp. & 0.000 & 0.000 & 0.003 \\
\hline Pleosporales sp. & 0.000 & 0.000 & 0.002 \\
\hline Talaromyces aculeatus & 0.002 & 0.000 & 0.004 \\
\hline Teratosphaeriaceae sp. & 0.000 & 0.000 & 0.002 \\
\hline Tremellales sp. & 0.000 & 0.000 & 0.003 \\
\hline Pichia fermentans & 0.081 & 0.022 & 0.017 \\
\hline
\end{tabular}

${ }^{1} \mathrm{M} 1=$ kefir grains $(2 \%) ; \mathrm{M} 2=$ kefir grains $(2 \%)+$ Saccharomyces cerevisiae BOF; M3 = kefir grains $(2 \%)+$ Saccharomyces cerevisiae KU200284. 


\section{ACKNOWLEDGMENTS}

This research was supported by the Korea Institute of Planning and Evaluation for Technology in Food, Agriculture and Forestry (IPET) through High Valueadded Food Technology Development Program, funded by the Ministry of Agriculture, Food and Rural Affairs (MAFRA; \#314073-03) and a grant from Korea Food Research Institute (Wanju, Korea; Project no. E0170700-03).

\section{REFERENCES}

Caporaso, J. G., J. Kuczynski, J. Stombaugh, K. Bittinger, F. D. Bushman, E. K. Costello, N. Fierer, A. G. Pena, J. K. Goodrich, J. I. Gordon, and G. A. Huttley. 2010. QIIME allows analysis of highthroughput community sequencing data. Nat. Methods 7:335-336.

Fakruddin, M., M. N. Hossain, and M. M. Ahmed. 2017. Antimicrobial and antioxidant activities of Saccharomyces cerevisiae IFST062013, a potential probiotic. BMC Complement. Altern. Med. 17:64.

Farnworth, E. R. 2005. Kefir-A complex probiotic. Food Science and Technology Bulletin: Functional Foods 2:1-17.

Garofalo, C., A. Osimani, V. Milanovic, L. Aquilanti, F. De Filippis, G. Stellato, S. Di Mauro, B. Turchetti, P. Buzzini, D. Ercolini, and F. Clementi. 2015. Bacteria and yeast microbiota in milk kefir grains from different Italian regions. Food Microbiol. 49:123-133.

Hong, J. Y., S. H. Son, S. P. Hong, S. H. Yi, N. K. Lee, and H. D. Paik. 2019. Production of $\beta$-glucan, and glutathione derivatives by probiotic Saccharomyces cerevisiae isolated from cucumber jangajji. Lebensm. Wiss. Technol. 100:114-118.

Kabak, B., and A. D. W. Dobson. 2011. An introduction to the traditional fermented foods and beverages of Turkey. Crit. Rev. Food Sci. Nutr. 51:248-260.

Kim, D. H., D. Jeong, I. B. Kang, H. W. Lim, Y. J. Cho, and K. H. Seo. 2019. Modulation of the intestinal microbiota of dogs by kefir as a functional dairy product. J. Dairy Sci. 102:3903-3911.

Kim, J. A., and M. S. Cho. 2009. Quality changes of immature green cherry tomato pickles with different concentration of soy sauce and soaking temperature during storage. J. Korean Soc. Food Cult. 24:295-307.

Koh, W. Y., U. Utra, A. Rosma, M. E. Effarizah, W. I. W. Rosli, and Y. H. Park. 2017. Development of a novel fermented pumpkin- based beverage inoculated with water kefir grains: A response surface methodology approach. Food Sci. Biotechnol. 27:525-535.

Lee, N. K., J. Y. Hong, S. H. Yi, S. P. Hong, J. E. Lee, and H. D. Paik. 2019. Bioactive compounds of probiotic Saccharomyces cerevisiae strains isolated from cucumber jangajji. J. Funct. Foods $58: 324-329$.

Leite, A. M. O., D. C. A. Leite, E. M. Del Aguilta, T. S. Alvares, R. S. Peixoto, M. A. Miguel, J. T. Silva, and V. M. F. Paschoalin. 2013 Microbiological and chemical characteristics of Brazilian kefir during fermentation and storage processes. J. Dairy Sci. 96:4149-4159.

Leuschner, R. G. K., T. P. Robinson, M. Hugas, P. S. Cocconcelli, F. Richard-Forget, G. Kleine, T. R. Licht, C. Nguyen-The, A. Querol, M. Richardson, J. E. Suarez, U. Thrane, J. M. Vlak, and A. von Wright. 2010. Qualified presumption of safety 342 (QPS): A generic risk assessment approach for biological agents notified to the European Food Safety Authority (EFSA). Trends Food Sci. Technol. 21:425-435.

Marshall, V. M., and W. M. Cole. 1985. Methods for making kefir and fermented milks based on kefir. J. Dairy Res. 52:451-456.

Nalbantoglu, U., A. Cakar, H. Dogan, N. Abaci, D. Ustek, K. Sayood, and H. Can. 2014. Metagenomic analysis of the microbial community in kefir grains. Food Microbiol. 41:42-51.

Ni, H. J., Q. H. Bao, T. S. Sun, X. Chen, and H. P. Zhang. 2007. Identification and biodiversity of yeasts isolated from koumiss in Xinjiang of China. Wei Sheng Wu Xue Bao 47:578-582.

Prado, M. R., L. M. Blandón, L. P. S. Vandenberghe, C. Rodrigues, G. R. Castro, V. Thomaz-Soccol, and C. R. Soccol. 2015. Milk kefir: Composition, microbial cultures, biological activities, and related products. Front. Microbiol. 6:1177.

Wang, S. Y., K. N. Chen, Y. M. Lo, M. L. Chiang, H. C. Chen, J. R. Liu, and M. J. Chen. 2012. Investigation of microorganisms involved in biosynthesis of the kefir grain. Food Microbiol. $32: 274-285$.

Wszolek, M., A. Y. Tamime, D. D. Muir, and M. N. I. Barclay. 2001. Properties of kefir made in Scotland and Poland using bovine, caprine and ovine milk with different starter cultures. Lebensm. Wiss. Technol. 34:251-261.

Zhang, D. D., J. L. Liu, T. M. Jiang, L. Li, G. Z. Fang, Y. P. Liu, and L. J. Chen. 2017. Influence of Kluyveromyces marxianus on proteins, peptides, and amino acids in Lactobacillus-fermented milk. Food Sci. Biotechnol. 26:739-748.

\section{ORCIDS}

Hyun-Dong Paik @ https://orcid.org/0000-0002-5131-3299 